\title{
The Search for Neutrinos from TXS 0506+056 with the ANTARES Telescope
}

\author{
Giulia Illuminati ${ }^{1, *}$ on behalf of the ANTARES Collaboration \\ ${ }^{1}$ IFIC - Instituto de Física Corpuscular (CSIC - Universitat de València) c/ Catedrático José Beltrán, 2 \\ E-46980 Paterna, Valencia, Spain
}

\begin{abstract}
A high-energy neutrino, IC170922A, detected by the IceCube experiment was found to be positionally coincident with the direction of a known blazar, TXS 0506+056, observed in $\gamma$-rays and at other wavelengths of the electromagnetic spectrum. Here, we present the results of three different searches for neutrino candidates, associated either with the IC170922A event or with the TXS 0506+056 blazar, performed by the ANTARES Collaboration. The three searches, an online follow-up of the IceCube alert, a standard time-integrated point-source analysis and a time-dependent search, yielded no significant observation. Consequently, 90\% C.L. upper limits on the one-flavour neutrino flux and fluence have been derived.
\end{abstract}

\section{Introduction}

On 22 September 2017, the IceCube Collaboration detected a high-energy (HE) neutrinoinduced muon track, with high probability of being of astrophysical origin [1]. The neutrinocandidate event, IC170922A, was selected by the Extremely High Energy (EHE) online event filter and reported through a Gamma-ray Coordinates Network (GCN) Circular [2]. It was soon found that IC170922A was coincident in direction and time with a gamma-ray flare from the blazar TXS 0506+056 [3]. Triggered by this association, the IceCube Collaboration performed a search for clustering in time at the position of TXS 0506+056 assuming two different generic profile shapes: a Gaussian-shaped time window and a box-shaped time window [1]. The analysis yielded a $3.5 \sigma$ evidence for an excess of high-energy neutrino events, with respect to atmospheric backgrounds, at the position of the blazar between September 2014 and March 2015. Prompted by these findings, three different analyses were performed using data of the ANTARES telescope [4] to investigate the location of the IceCube HE event and of the blazar. The three searches are presented in this contribution and in detail in [5]. In Section 2, the search for neutrino candidates in the ANTARES online data stream, triggered by the GCN circular, is described. In Section 3, the result of the ANTARES standard point-source analysis at the position of the blazar, motivated by the potential association between IC170922A and TXS 0506+56, is presented. The ANTARES time-dependent analysis on the bursting period reported by the IceCube Collaboration is described in Section 4. The conclusions are summarised in Section 5.

\footnotetext{
*e-mail: giulia.illuminati@ific.uv.es
} 


\section{Online searches}

Soon after the identification of the IceCube event by means of the EHE track event selection, a GCN Circular was issued. Follow-up searches were encouraged by the IceCube Collaboration to help identifying a possible astrophysical source at the same direction as the event. Triggered by this alert, the ANTARES online data stream was scrutinised to find HE neutrino candidates from the originally communicated location of IC170922A, i.e. RA $=77.43^{\circ}{ }_{-0.80^{\circ}}$ and $\delta=5.72^{\circ}{ }_{-0.40^{\circ}}^{+0.70^{\circ}}$. For neutrino energies below $100 \mathrm{TeV}$, ANTARES has competitive sensitivity to this position in the sky. At the location of the ANTARES detector, the position of the IceCube event corresponds to a direction of $14.2^{\circ}$ below the horizon. Therefore, neutrino events from the direction of IC170922A would be detected as upgoing in the ANTARES telescope, as shown in Figure 1.

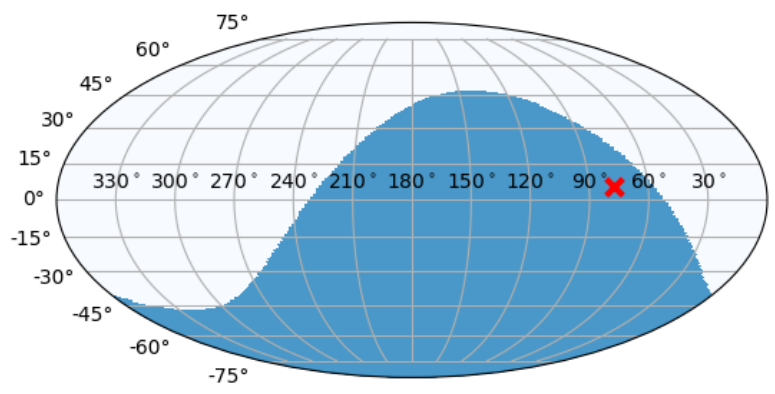

Figure 1. Visibility map for the ANTARES detector of IC170922A (represented by the red marker) in equatorial coordinates. The sky regions below and above the horizon at the alert time are shown in blue and white, respectively. Events that originate from the blue (white) region will be seen as upgoing (downgoing) in the detector frame.

A fast algorithm [6], which uses an idealised detector geometry and no information about the dynamical positioning calibration, was employed to scan the online data-set and select up-going neutrino track candidates. Muon neutrino events are reconstructed with a median angular resolution better than $0.5^{\circ}$ at $10 \mathrm{TeV}$. Upgoing muon neutrino candidate events were searched for in two time-windows, namely $\pm 1 \mathrm{~h}$ and \pm 1 day, centred on the IceCube event time and in a cone of $3^{\circ}$ centred on the event coordinates. No ANTARES event was recorded in any of the two considered time-windows. The non-detection within $2 \mathrm{~h}$ from the IceCube event was used to provide a preliminary constraint on the neutrino fluence assuming a pointsource emitting with an unbroken power-law spectrum $E^{-\gamma}$. For a specral index $\gamma=2.0$, the $90 \%$ C.L. fluence upper limit is $15 \mathrm{GeV} \mathrm{cm}^{-2}$, integrated over the energy range $3.3 \mathrm{TeV}-3.4$ $\mathrm{PeV}$ (the range corresponding to 5-95\% of the detectable flux). For $\gamma=2.5$, the 90\% C.L. fluence upper limit is $34 \mathrm{GeV} \mathrm{cm}^{-2}$, integrated in the $450 \mathrm{GeV}-280 \mathrm{TeV}$ energy range.

\section{Time-integrated search}

Motivated by the potential association between IC170922A and TXS 0506+56, the ANTARES standard candidate-list point-source method [7] was applied to investigate the location of the blazar. In this approach, the directions of a predefined list of neutrino source candidates are scrutinised to look for an excess of events using a maximum likelihood ratio 
method. The likelihood exploits the energy and directional information of each event to distinguish signal-like clusters from clusters produced by random agglomerations of background events.

The source was first added to the list of 106 pre-selected sources analysed in a previous ANTARES work [7] and scrutinised using data recorded in 2424 days of livetime (from the beginning of 2007 to the end of 2015) including both track-like and shower-like events. Out of the 107 investigated sources, the cluster at the location of the blazar was found to be the third most significant one, with a number of fitted signal events $\mu_{\text {sig }}=1.03$ and a pre-trial p-value of $2.6 \%$ (assuming an unbroken power-law spectrum $E^{-2.0}$ ). When accounting for the fact that multiple candidates are analysed, the post-trial p-value for TXS $0506+056$ is $87 \%$.

Once the fully calibrated data collected by the ANTARES detector during 2016 and 2017 became available, a standard point-source search limited to the position of TXS 0506+056 was performed using the whole available data-set (3136 days of livetime). After the inclusion of the 2016-2017 data, the number of fitted signal events, $\mu_{\text {sig }}$, remains 1.03 with an associated p-value of $3.4 \%$. There is one track-like event mostly influencing the fit (see Fig. 2). It occurred on December 12, 2013 (MJD: 56638.70832) and is located within $1 \sigma$ from the source position. The value of the energy estimator, $\rho$, for this event is such that only $9 \%$ of the neutrino candidates inducing a track have a larger value.

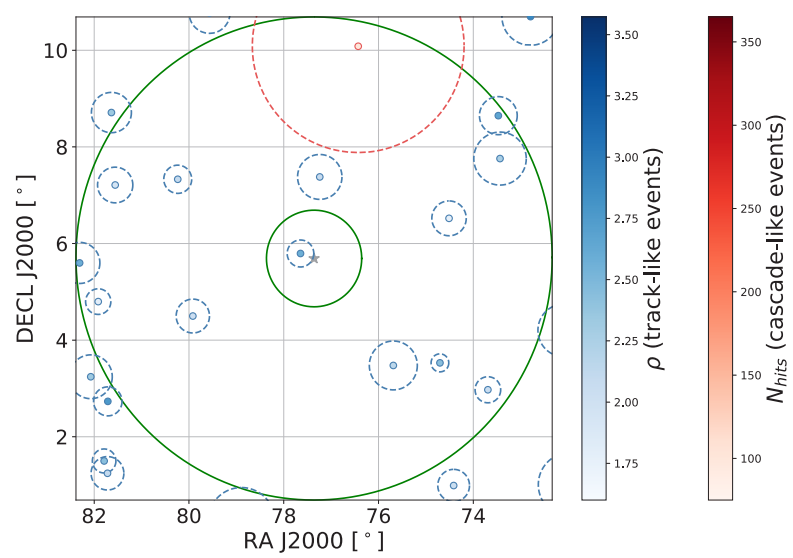

Figure 2. Distribution of the ANTARES events collected in 3136 days of livetime (2007-2017 period) in the (RA, $\delta$ ) coordinates around the position of TXS $0506+056$. The inner (outer) green line indicates the one (five) degree distance from the source position, shown as a gray star. The red point denotes a shower-like event, whereas the blue points indicate track-like events. The dashed circles around the events indicate the angular error estimate. Different tones of red and blue correspond to the values assumed by the energy estimators - the number of hits for shower-like events and the $\rho$ parameter for track-like events—as shown in the legend. Refer to [7] for further details on the energy estimators.

Using the total livetime of 3136 days, the $90 \%$ C.L. upper limits on the flux normalization factor at the energy of $100 \mathrm{TeV}, \Phi_{100}^{90 \% \mathrm{TeV}}$, assuming a steady neutrino source emitting with unbroken power-law spectra $E^{-2.0}\left(E^{-2.3}\right)\left[E^{-2.5}\right]$, were set to $1.6(1.4)[1.0] \times 10^{-18} \mathrm{GeV}^{-1} \mathrm{~cm}^{-2}$ $\mathrm{s}^{-1}$. 


\section{Time-dependent searches}

After the identification of an excess of neutrinos in the IceCube data localised in a restricted time window, a time-dependent analysis was performed assumuming the two time profiles provided by the IceCube Collaboration. The ANTARES time-dependent method uses the same formulation of the likelihood as the one employed in the time-integrated analysis, with the addition of a time-dependent term. The first time profile (referred to as Gaussian flare) is described by a Gaussian signal centred on MJD 57004 and with standard deviation $\sigma=$ 55.0 days. Neutrinos were searched for in a period $\pm 5 \sigma$ wide, corresponding to 550 flaring days. The second one (referred to as box flare) assumes a box-shaped flare starting at MJD 56937.81 and ending at MJD 57096.21, corresponding to 158.40 flaring days.

The search in neither of the considered flares yielded a significant observation. Within $2^{\circ}$ from the source, 13 events have been found in data, with 10 being the expected number of background events during the analysed period. None of the signal events was detected within either of the two considered flaring periods.

As no significant evidence of cosmic neutrino was observed, 90\% C.L. upper limits have been derived for the neutrino flux. For the Gaussian-shaped period and unbroken energy spectra $E^{-2.0}\left(E^{-2.1}\right)\left[E^{-2.2}\right]$, the limits correspond to normalization factors of $\Phi_{100 \mathrm{TeV}}^{90 \%}=4.6(4.4)[4.2] \times 10^{-18} \mathrm{GeV}^{-1} \mathrm{~cm}^{-2} \mathrm{~s}^{-1}$. The energy range containing the 5-95\% of the detectable flux is 2.0 (1.3) [1.0] TeV - 3.2 (1.6) [1.0] PeV. The limits on the flux normalization factors for box-shaped period are a factor 3.3 higher.

\section{Conclusions}

Motivated by the detection of the HE neutrino event by the IceCube experiment, its potential association with the known blazar and the results of the time-dependent analysis performed by the IceCube Collaboration, three different searches for neutrino candidates have been performed by the ANTARES Collaboration. As no significant excess over the expected background was observed, $90 \%$ C.L. upper limits on the one-flavour neutrino flux and fluence have been derived.

We gratefully acknowledge the financial support of Ministerio de Economía y Competitividad (MINECO): Plan Estatal de Investigación (ref. FPA2015- 65150-C3-1-P), (MINECO/FEDER)), Severo Ochoa Centre of Excellence (MINECO), and Prometeo and Grisolía programs (Generalitat Valenciana), Spain.

\section{References}

[1] M. G. Aartsen et al. (IceCube Collaboration), Science 361 no.6398, 147-151 (2018)

[2] IceCube Collaboration, GCN circular 21916; https://gcn.gsfc. nasa.gov/gcn3/21916.gen3 (2017)

[3] The IceCube Collaboration, Fermi-LAT, MAGIC, AGILE, ASAS-SN, HAWC, H.E.S.S., INTEGRAL, Kanata, Kiso, Kapteyn, Liverpool Telescope, Subaru, Swift/NuSTAR, VERITAS, VLA/17B-403 teams, Science 361 no.6398, eaat1378 (2018)

[4] M. Ageron et al., (ANTARES Collaboration), Nucl.Instrum.Meth. A656, 11 (2011)

[5] A. Albert et al. (ANTARES Collaboration), Astrophys.J. 863 no.2, L30 (2018)

[6] S. Adrian-Martinez et al. (ANTARES Collaboration), JCAP 1602 no.02, 062 (2016)

[7] A. Albert et al. (ANTARES Collaboration), Phys.Rev. D96 no.8, 082001 (2017) 